\title{
Nanochannel-confined growth of crystallographically orientated perovskite nanowire arrays for polarization-sensitive photodetector application
}

\author{
Rui Huang ${ }^{1}$, Di-Hua Lin ${ }^{1}$, Jia-Yin Liu ${ }^{1}$, Chun-Yan $\mathrm{Wu}^{1^{*}}, \mathrm{Di} \mathrm{Wu}^{2}$ and Lin-Bao Luo ${ }^{1^{*}}$
}

\begin{abstract}
Highly ordered perovskite nanowire (PNW) arrays are important building blocks for potential application in integrated optoelectronic devices due to their unique properties. Herein, a recordable digital versatile disk-assisted nanochannel-confined growth (NCG) strategy was developed for large-scale growth of different kinds of PNW arrays with preferentially crystallographic orientation on various substrates. Photodetector constructed from $\mathrm{MAPbI}_{3} \mathrm{NW}$ arrays exhibits prominent photoresponse properties with a responsivity of $20.56 \mathrm{~A} \mathrm{~W}^{-1}$ and specific detectivity of $4.73 \times$ $10^{12}$ Jones, respectively. What is more, the photodetector can function as a polarization-sensitive photodetector due to the crystallographic orientation of the one-dimensional PNW arrays, with a polarization ratio of 2.2 . The proposed NCG strategy provides a cost-efficient and effective method for the fabrication of high-quality PNW arrays with potential applications in future integrated devices and systems.
\end{abstract}

Keywords: nanochannel-confined growth, one-dimensional nanostructures, optoelectronic devices, polarization photodetection, template

\section{INTRODUCTION}

Metal halide perovskites, including organic-inorganic hybrid perovskites $\left(\mathrm{MAPbX}_{3}, \mathrm{FAPbX}_{3}\right.$, etc., $\mathrm{MA}=$ methylammonium, $\mathrm{FA}=$ formamidinium, $\mathrm{X}=\mathrm{Cl}, \mathrm{Br}, \mathrm{I}$ ) and allinorganic perovskites $\left(\mathrm{CsPbX}_{3}, \mathrm{Cs}_{3} \mathrm{Cu}_{2} \mathrm{I}_{5}\right.$, etc. $)$ have emerged as important candidates for assembly of highperformance optoelectronic devices such as light-emitting devices [1-3], photovoltaics [4-7], and photodetectors [8-11], due to their adjustable bandgap, large optical absorption coefficient, high carrier mobility, and long carrier diffusion length [12-16]. Compared with their film or bulk counterparts, one-dimensional (1D) perovskite nanowires (PNWs) often display attractive physical and optoelectronic merits, such as excellent mechanical stability [17], optical anisotropy in axial and radial directions $[18,19]$, and improved charge carrier transportation [20]. For these reasons, 1D PNWs have recently attracted increasing research interests for their promising applications in ultrasensitive photodetectors $[21,22]$.

Owing to the vulnerability of perovskite materials to humidity and other polar or protic solvents, it is impossible to fabricate PNW arrays via conventional photolithography process, followed by harsh developing and etching process in base or organic solutions [23]. Other direct patterning technologies, such as laser direct-write (LDW) method [24], focused ion beam (FIB) etching [25], and inkjet printing technology [26], are indeed capable of fabricating well-defined perovskite arrays. However, the expensive apparatuses as well as the timeconsuming process constitute a bottleneck for their practical application. As optional alternatives, some solution-processed strategies have proved to be low-cost and effective patterning approaches [27-29], and to date a number of approaches, such as evaporation-induced selfassembly [18,30], blade-coating method [31] and template confined/induced self-assembly $[19,32,33]$, have been developed for the fabrication of various perovskite nanowire (NW)/microwire (MW) arrays. For example, Deng et al. [31] achieved the fabrication of high-quality single-crystalline $\mathrm{MAPbI}_{3} \mathrm{MW}$ arrays by a straightforward blade-coating method. The $\mathrm{MAPbI}_{3} \mathrm{MW}$ arraysbased photodetector exhibited a high responsivity of $13.57 \mathrm{~A} \mathrm{~W}^{-1}$ as well as excellent flexibility and robust

\footnotetext{
${ }^{1}$ School of Electronic Science and Applied Physics, Hefei University of Technology, Hefei 230009, China

${ }^{2}$ School of Physics and Microelectronics, Zhengzhou University, Zhengzhou 450052, China

* Corresponding authors (emails: cywu@hfut.edu.cn (Wu CY); luolb@hfut.edu.cn (Luo LB))
} 
bending stability. What is more, well-aligned PNW (e.g., $(\mathrm{BA})_{2}(\mathrm{MA})_{3} \mathrm{~Pb}_{4} \mathrm{I}_{13} \quad(\mathrm{BA}=$ butylacrylate $)$ and $\left.\mathrm{CsPbr}_{3}\right)$ arrays can also be fabricated through an asymmetricwettability micropillar template. Photodetector made of the $(\mathrm{BA})_{2}(\mathrm{MA})_{3} \mathrm{~Pb}_{4} \mathrm{I}_{13}$ exhibits outstanding photoresponse characteristics, with a responsivity exceeding $1.5 \times 10^{4} \mathrm{~A} \mathrm{~W}^{-1}$ and specific detectivity larger than $7 \times$ $10^{15}$ Jones [10]. Although the above methods have indeed proved to be effective for the fabrication of PNW arrays, it is undeniable that these methods still suffer from some shortcomings, such as the poor morphology and position control, selective modification with expensive organic molecules or complicated processes including photolithography and etching.

To solve the above-mentioned dilemma, we herein proposed a recordable digital versatile disk (DVD-R)assisted nanochannel-confined growth (NCG) strategy for large-scale growth of crystallographically orientated nanowire arrays. By taking this strategy, various 1D PNW arrays on different substrates with controllable width (330-660 nm) have been obtained. Further device analysis reveals that photodetector composed of $\mathrm{MAPbI}_{3} \mathrm{NW}$ arrays has displayed a responsivity of $20.56 \mathrm{~A} \mathrm{~W}^{-1}$ and specific detectivity of $4.73 \times 10^{12}$ Jones, which are better than the values of most PNW devices derived from other methods. It is also found that the PNW device is capable of detecting polarized light, with a polarization ratio of 2.2. We believe this work provides a universal approach for the facile and large-scale growth of PNW arrays with preferentially crystallographic orientation, which may have potential applications in future integrated optoelectronic devices.

\section{EXPERIMENTAL SECTION}

\section{Fabrication of nanochannel-confined template}

The commercial $16 \times$ speed 4.7 GB DVD-R used in this work was purchased from Philips Company. The disk was discomposed from the cross-section and the polycarbonate protective layer (layer I) and the metal film reflective layer (layer II) were removed using a blade. Afterwards, the dye recording layer (layer III) was rinsed with ethanol to remove the dye and then blow dried by $\mathrm{N}_{2}$ flow. The left polycarbonate layer with submicron linear track (layer IV) would be used as the DVD-R master template. A mixture of polydimethylsiloxanes (PDMS) precursor and cross-linker (Sylgard 184, Dow Corning, with a 10:1 weight ratio) was cast onto the master template. After being cured at $60^{\circ} \mathrm{C}$ for $4 \mathrm{~h}$ and peeled off, a PDMS template with parallel nanoscale grooves was ob- tained, which was cut into $2 \times 2 \mathrm{~cm}^{2}$ prior to use.

\section{Fabrication of well-aligned PNWPNW arrays}

Well-aligned PNW arrays were prepared through the DVD-R-assisted NCG method. The perovskite precursor solutions were prepared by dissolving a 1:1 molar ratio of MAX (or FAX) and $\mathrm{PbX}_{2}(\mathrm{X}=\mathrm{Cl}, \mathrm{Br}, \mathrm{I})$ in $N$-dimethylformamide (DMF, >99.9\%), a 1:1 molar ratio of CsI and $\mathrm{PbI}_{2}$ (a 3:2 molar ratio of CsI and $\mathrm{CuI}$ for $\left.\mathrm{Cs}_{3} \mathrm{Cu}_{2} \mathrm{I}_{5}\right)$ in a mixed solvent of DMF and dimethyl sulfoxide (DMSO, >99.9\%) at a volume ratio of $1: 1$, followed by stirring at $60^{\circ} \mathrm{C}$ for $12 \mathrm{~h}$. In a typical process for preparing the $\mathrm{PNW}$ arrays, the pre-cleaned substrates (e.g. $\mathrm{SiO}_{2} / \mathrm{Si}$, glass, polypropylene (PP) and polyethylene terephthalate (PET)) were firstly treated by oxygen plasma (Harrick PDC-32G-2, $18 \mathrm{~W}$ ) for 5 min to obtain hydrophilic surfaces. Five microliter of precursor solution was dropped onto the hydrophilic substrate and a thin precursor film will be formed on the surface immediately due to the wetting of the solution on the hydrophilic substrate. A piece of PDMS template was then gently imprinted on the perovskite precursor solution-coated substrate under a moderate pressure (about $10 \mathrm{kPa}$ ) provided by the holding equipment shown in Fig. S1. The substrate with PDMS template was kept under ambient conditions for $12 \mathrm{~h}$ so that the solvent can be gradually evaporated (the solvent containing DMSO was kept at $70^{\circ} \mathrm{C}$ for $6 \mathrm{~h}$ in a dry oven). The PDMS template was peeled off after the solvent was completely evaporated. Well aligned PNW arrays were formed on the substrate, which were further annealed at $90^{\circ} \mathrm{C}$ for $15 \mathrm{~min}$ for better crystallization.

\section{Structural analysis and device characterization}

The compositions and morphologies of the PNW arrays were characterized by X-ray diffraction (XRD, X-Pert PRO MPD, PANalytical) and field emission scanning electron microscopy (FESEM, Hitachi SU8020). The absorption spectrum was recorded on an ultraviolet-visiblenear-infrared (UV-NIR) spectrophotometer (Cary 5000). The surface morphology and the topographic profiles of the DVD-R master template, the PDMS template and the obtained PNW arrays were observed by atomic force microscope (AFM, Dimension Icon, Bruker).

For the optoelectronic characterization of the PNW arrays, parallel $\mathrm{Au}(50 \mathrm{~nm})$ electrodes with a channel width of $18 \mu \mathrm{m}$ were deposited onto the opposite ends of the NWs by electron beam evaporation through a labbuilt shadow mask. Optoelectronic characterization was conducted on a semiconductor characterization system 
(Keithley 4200-SCS) equipped with a monochromator (SP 2150, Princeton Co.) under ambient conditions at room temperature. The laser diode with a wavelength of $520 \mathrm{~nm}$ (LP520-SF15) was used as light sources and the power intensity was carefully calibrated using a power meter (Thorlabs GmbH., PM 100D) before measurement.

\section{RESULTS AND DISCUSSION}

In this strategy, a piece of commercial DVD-R with nanoscale tracks was used as the master template (the internal structure is schematically shown in Fig. S2). The PDMS template with nanochannels was formed by casting a mixture of PDMS precursor and cross-linker onto the DVD-R master template and therefore decently replicating the nanostructures in the DVD-R master template. The detailed fabrication of well-aligned PNW arrays can be learned from Fig. 1a and the Experimental Section. As depicted in the steps II-III of Fig. 1a, when the PDMS template with nanoscale grooves was gently imprinted onto the substrate, the solution on the substrate would be redistributed and confined in the na- nochannels formed between the PDMS template and the substrate. The triple-phase contact line was formed at the ends of the nanochannels, where the nucleation of perovskite molecules would occur due to the high surface energy [34]. In the steps IV-V, with the slow evaporation of the solvent, more perovskite molecules in the solution would gradually precipitate out, which would then selfassemble preferentially along the nanochannels, leading to well-aligned PNW arrays [31].

In this study, the decent replication of the nanostructures in the DVD-R master template is vitally important for the successful fabrication of well-defined PNW arrays. To evaluate the effectiveness of the DVD-Rassisted NCG strategy, the surface morphologies of the DVD-R master template and the obtained PDMS template were compared. Fig. $1 b$ and $c$ present the AFM images of the DVD-R master template and the PDMS template, where periodic structures with uniform dimension can be easily observed. As a matter of fact, these periodic gratings are also reflected by the distinct color on both DVD-R master template and the NCG template

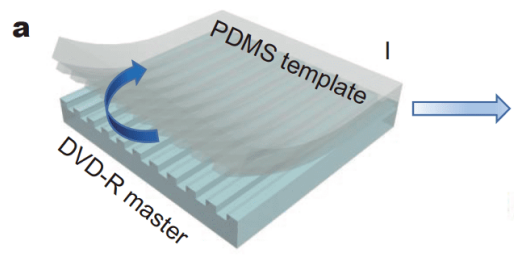

Fabrication of NCG template

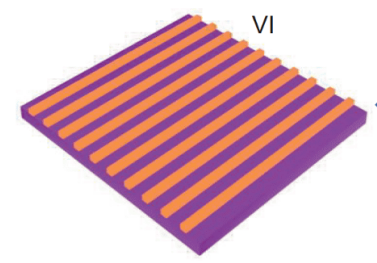

Well-aligned PNW arrays

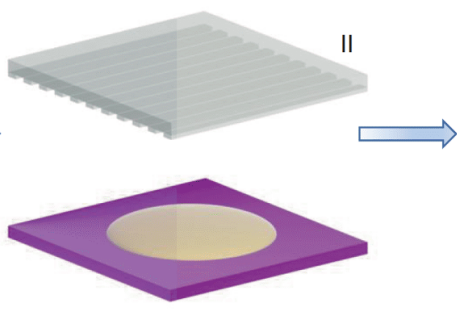

Dropping precursor solution
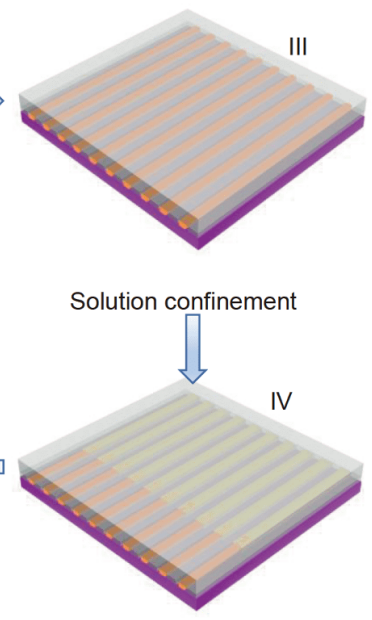

Confined growth

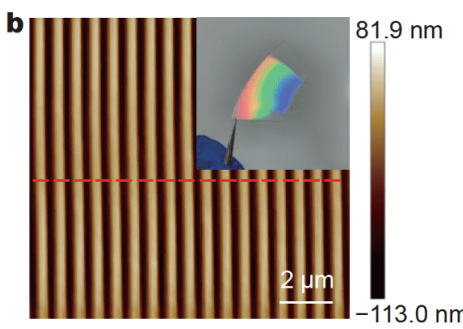

Growth of 1D array

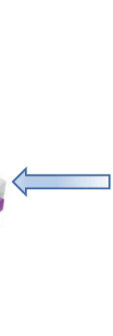

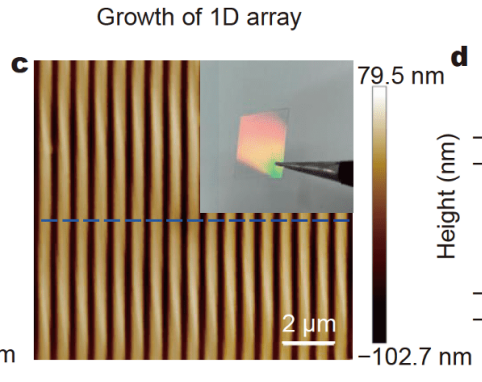

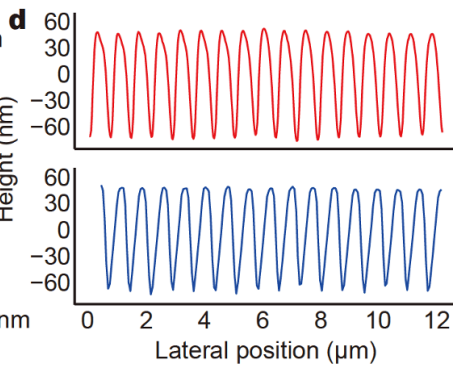

Figure 1 Fabrication of the PNW arrays through a DVD-R-assisted NCG strategy. (a) Schematic illustration of the preparation process for the NCG strategy. AFM images of (b) a representative DVD-R master template and (c) the prepared PDMS template. The insets show the corresponding optical photographs. (d) Topographic profiles across the dotted line in panels (b) and (c). 
under sunlight illumination, which should be ascribed to the light-matter interaction (the insets of Fig. 1b, c). According to the corresponding topographic profiles in Fig. 1d, the depth, full width at half maximum (FWHM) and period of the NCG template is about 110, 450 and $760 \mathrm{~nm}$, respectively, which are very close to those of the master template $(120,470$ and $760 \mathrm{~nm})$, indicating that the NCG template has replicated the nanostructures of the master template.

The PNW arrays derived from the NCG strategy are characterized by highly ordered arrangement of the nanostructures with nearly identical dimension and morphology. Taking $\mathrm{MAPbI}_{3}$ for example, when using $0.25 \mathrm{~mol} \mathrm{~L}^{-1}$ perovskite solution as precursor, very uniform and well-aligned NW arrays can be observed in the whole field of view (Fig. 2a). The large-scale and homogeneous deposition of ordered PNW arrays on the $1 \times 1 \mathrm{~cm}^{2}$ substrate is confirmed by the optical photograph shown in the inset of Fig. 2a, which exhibits shiny green because of light interference of the well-aligned nanogratings [32]. Further high-magnification SEM images (Fig. 2b, c) reveal that the obtained PNWs have uniform morphology and smooth surface without any apparent defect or distortion, which means that these samples are superior to those previously prepared by the imprinted method [17]. The width and interval of the PNWs is about 430 and $330 \mathrm{~nm}$, respectively, which are smaller than those of the PDMS template due to shrinkage in volume after evaporation. However, the period of the nanograting structures is consistent with that of the nanochannels in the PDMS template $(760 \mathrm{~nm})$, verifying the confined growth of PNWs. The XRD pattern of the obtained PNW arrays in Fig. 2d exhibits only two remarkable diffraction peaks located at $14.27^{\circ}$ and $28.56^{\circ}$, due to (110) and (220) crystalline planes of $\mathrm{MAPbI}_{3}$, respectively, suggesting the good crystallinity and preferential orientation along the [110] direction [18]. Energy-dispersive X-ray spectroscopy (EDS) element mappings (Fig. 2e) reveal that both $\mathrm{Pb}$ and I elements are homogeneously distributed in an individual $\mathrm{MAPbI}_{3} \mathrm{NW}$. The atomic ratio of $\mathrm{I} / \mathrm{Pb}$ is calculated to be about 2.88 (see Fig. S3), which is very close to the stoichiometric ratio of $\mathrm{MAPbI}_{3} . \mathrm{Pb}$ or I element can hardly be clearly identified in the region between two adjacent nanowires, confirming the confined growth of PNW array. The AFM image and height profile in Fig. $2 \mathrm{f}$ show that all the NWs have a uniform height of about $65 \mathrm{~nm}$, which is lower than the depth of the nanochannels in the PDMS template. We believe this should be ascribed to the evaporation of the solvent confined in the nanochannels of the PDMS
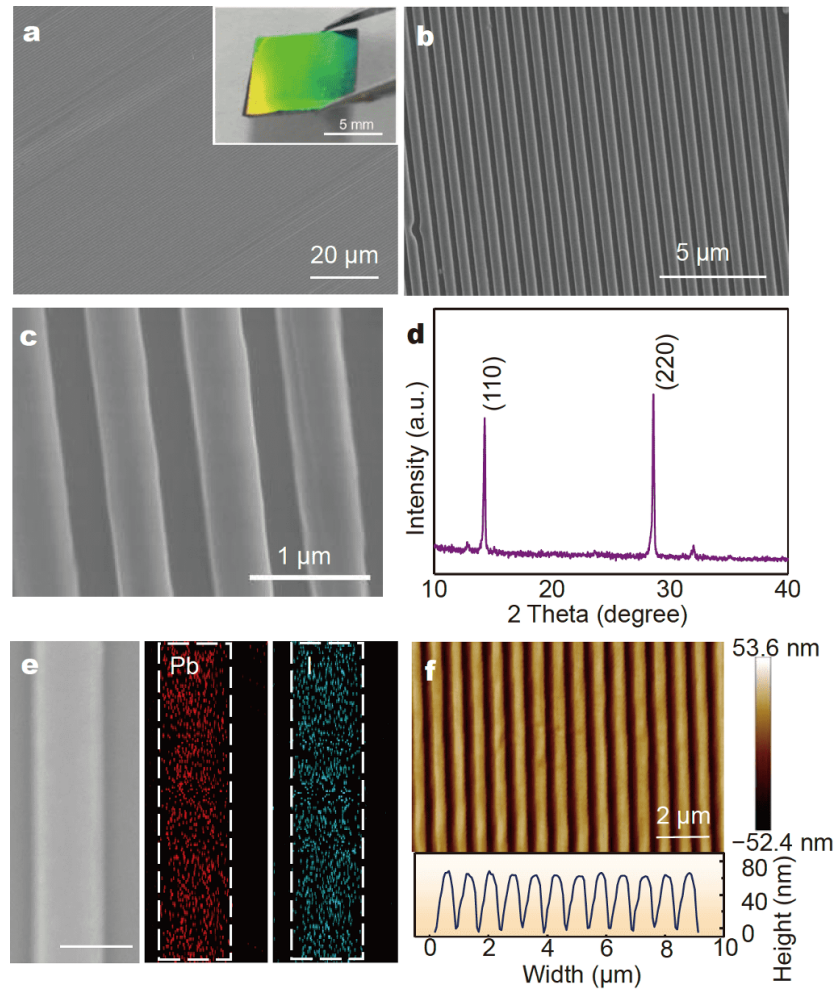

Figure 2 Characterizations of the PNW arrays. (a) Low-, (b) medium-, and (c) high-magnification SEM images of $\mathrm{MAPbI}_{3} \mathrm{NW}$ arrays on $\mathrm{SiO}_{2} /$ Si substrate. The inset in panel (a) exhibits the optical photograph of the $\mathrm{MAPbI}_{3} \mathrm{NW}$ arrays. (d) XRD pattern of the obtained $\mathrm{MAPbI}_{3} \mathrm{NW}$ arrays. (e) A magnified SEM image and corresponding EDS elemental mapping of a single $\mathrm{MAPbI}_{3} \mathrm{NW}$. The scale bar is $500 \mathrm{~nm}$. (f) AFM image (top panel) and the height profile (below panel) of the PNW arrays.

\section{template.}

It is noteworthy that the concentration of the precursor solution can directly determine the growth of the PNW arrays in the current DVD-R-assisted NCG strategy [35]. As illustrated in Fig. S4, while the period of PNW arrays remains to be about $760 \mathrm{~nm}$ for other concentrations of $\mathrm{MAPbI}_{3}$ precursor solution, the width and the morphology of the NWs vary greatly. For instance, when the concentration decreases from 0.5 to $0.125 \mathrm{~mol} \mathrm{~L}^{-1}$, the width of the NWs will decrease from 630 to $330 \mathrm{~nm}$ (see Fig. $54 a-c)$. This is reasonable as relatively small quantity of perovskite solute would be confined in the nanochannels at low concentrations. When the concentration of perovskite precursor solution further decreases to $0.03 \mathrm{~mol} \mathrm{~L}^{-1}$, discontinuous PNW arrays will be obtained due to the shortage of solute (see Fig. S4e). These results demonstrate that 1D PNWs with controllable width can be obtained by adjusting the concentration of the precursor solution. 
In addition to the above advantages in controlling the diameters of the PNWs, the present NCG strategy also allows the growth of various PNWs (e.g. $\mathrm{MAPbBr}_{3}$, $\mathrm{MAPbCl}_{3}, \mathrm{FAPbBr}_{3}, \mathrm{FAPbCl}_{3}$ and all-inorganic perovskites such as $\mathrm{CsPbI}_{3}$ and $\left.\mathrm{Cs}_{3} \mathrm{Cu}_{2} \mathrm{I}_{5}\right)$ on $\mathrm{SiO}_{2} / \mathrm{Si}$ substrate. The corresponding SEM images (Fig. 3a-f) and XRD patterns (Fig. S5) confirm that the as-obtained samples are all composed of well-aligned PNW arrays with good crystallinity and preferential crystallographic orientation [20,36-38]. Significantly, the PNW arrays can also be grown on other rigid and flexible substrates including glass, PP and PET, as long as these substrates are hydrophilic after surface treatment. Fig. 3g-i show the SEM images of the PNW arrays grown on different substrates, from which well-aligned NW arrays with smooth surface and uniform morphology are observed. Like the PNW arrays on $\mathrm{SiO}_{2} / \mathrm{Si}$ substrate, similar shiny color due to strong light interference can be observed for all the obtained PNW arrays, indicative of formation of large-area well-aligned nanograting structures. The excellent adaptability of this method for substrates stems from the good flexibility of PDMS material, which allows it to form intimate contact with the underlying substrate $[39,40]$. In addition, within the nanochannel, the slow volatilization of solvent can facilitate the gradual pre- cipitation and deposition of the perovskite molecules on the substrate, which is vitally important for the growth of high-quality PNW arrays.

Various studies have shown that high-quality, crystallographically orientated nanostructures may provide a direct axial transportation path for the charged carriers, which may facilitate their applications in optoelectronic devices $[20,41,42]$. In light of this, we then assembled photodetectors based on the well-aligned $\mathrm{MAPbI}_{3} \mathrm{PNW}$ arrays, in an effort to explore their potential applications for optoelectronic devices. Fig. 4a illustrates the device geometry of a typical photodetector based on $\mathrm{MAPbI}_{3}$ NW arrays and the optical photograph of a typical device with a channel width of $18 \mu \mathrm{m}$ (Fig. S6). It is seen that once the device was illuminated by $520 \mathrm{~nm}$ incident light, it exhibited remarkable photoresponse, with the photocurrent increasing monotonously as the incident light power density increased from $3.48 \mu \mathrm{W} \mathrm{cm}^{-2}$ to $1.45 \mathrm{~mW} \mathrm{~cm}^{-2}$. This evolution is reasonable since more photon-induced carriers would be generated at high incident light power densities. The dependence of the net photocurrent $\left(I_{\mathrm{ph}}=I_{\text {light }}-I_{\text {dark }}\right.$, where $I_{\text {light }}$ is the photocurrent and $I_{\text {dark }}$ is the dark current) on power density $(P)$ can be described by using a general power law $\left(I_{\mathrm{ph}} \propto P^{\theta}\right)$, where the exponent $\theta$ is the empirical value reflecting the

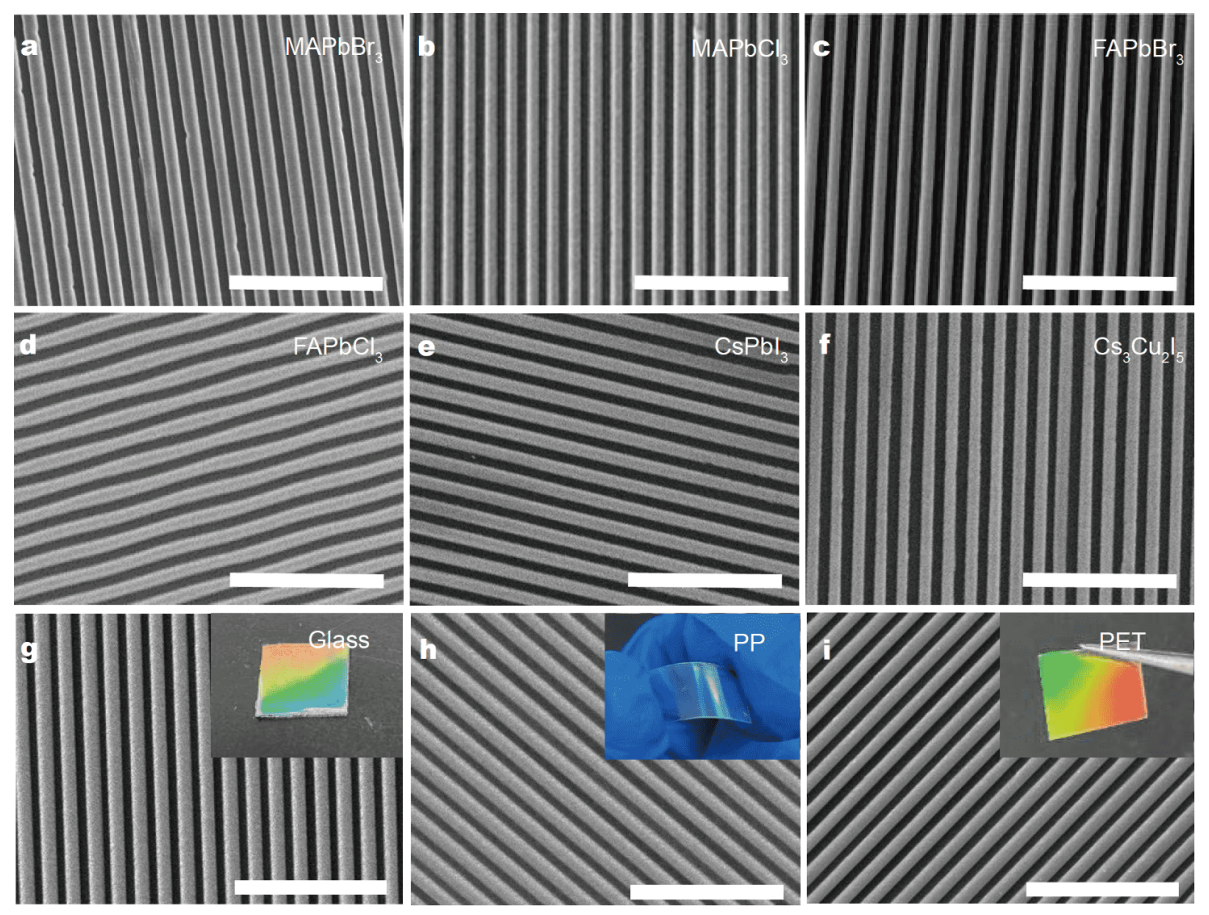

Figure 3 The universality of the NCG strategy. Different kinds of PNW arrays including (a) $\mathrm{MAPbBr}_{3}$, (b) $\mathrm{MAPbCl}_{3},(\mathrm{c}) \mathrm{FAPbBr}_{3},(\mathrm{~d}) \mathrm{FAPbCl}_{3}$, (e) $\mathrm{CsPbI}_{3}$ and (f) $\mathrm{Cs}_{3} \mathrm{Cu}_{2} \mathrm{I}_{5}$ deposited on $\mathrm{SiO}_{2} / \mathrm{Si}$ substrate and $\mathrm{MAPbI}_{3} \mathrm{NW}$ arrays deposited on (g) glass, (h) PP and (i) PET. The insets show the corresponding optical photographs of the PNW arrays in panels $(\mathrm{g}-\mathrm{i})$. The scale bars are $5 \mu \mathrm{m}$. 

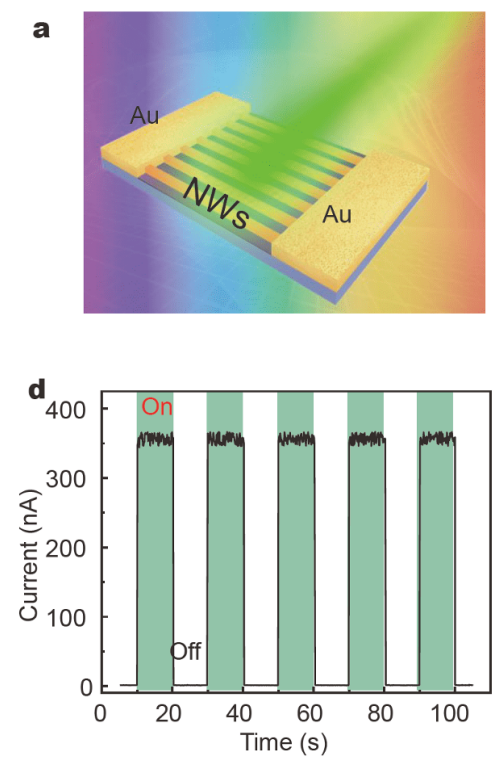
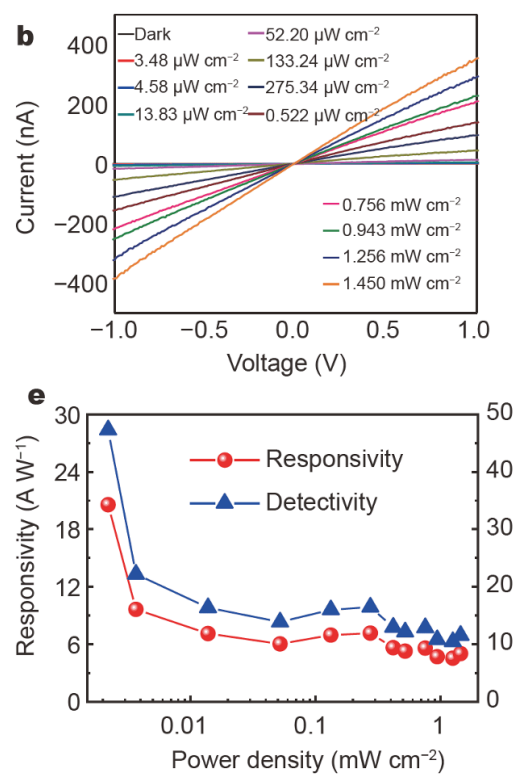
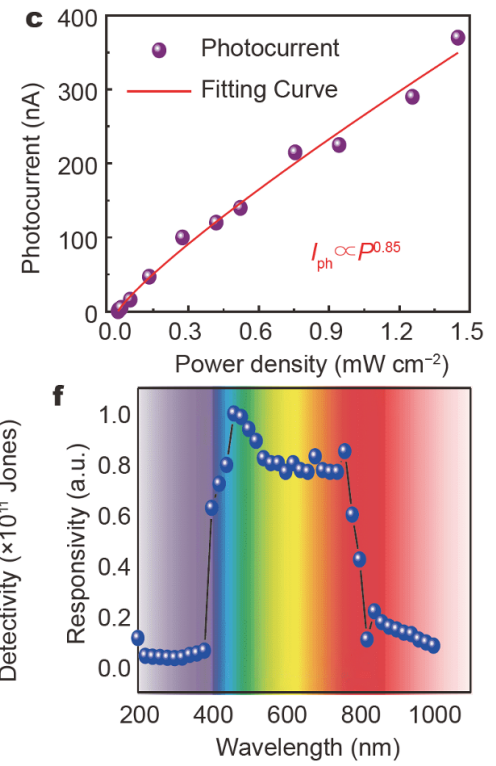

Figure 4 Optoelectronic properties of the PNW arrays. (a) Schematic illustration of the aligned $\mathrm{MAPbI}_{3} \mathrm{NW}$ arrays-based photodetector. (b) Current-voltage $(I-V)$ curves of a typical photodetector in dark and upon $520 \mathrm{~nm}$ illumination with varied incident light power densities from $3.48 \mu \mathrm{W} \mathrm{cm}{ }^{-2}$ to $1.45 \mathrm{~mW} \mathrm{~cm}^{-2}$. (c) Photocurrent as a function of the power density. (d) Time-dependent photoresponse of the device under $520 \mathrm{~nm}$ light illuminations $\left(1.45 \mathrm{~mW} \mathrm{~cm}^{-2}\right)$ at the bias voltage of $1 \mathrm{~V}$. (e) Dependence of responsivity and specific detectivity on the power density. (f) Spectral response of the device in the wavelength range of 200-1000 nm.

recombination of the photo-generated carriers and would be determined by the linear relationship of incident light power density and photocurrent (Fig. 4c) [43]. Fitting the experimental result leads to a non-integral $\theta$ of 0.85 , signifying the complicated process of electron-hole generation, trapping and recombination loss during the photodetection process $[43,44]$. Specifically, the device displays steady and repeatable switching characteristics between the high- and low-resistance states (Fig. 4d). The dark current is around $0.3 \mathrm{nA}$ while the current rises sharply to $350 \mathrm{nA}$ upon light illumination, yielding a high $I_{\text {light }} / I_{\text {dark }}$ ratio of about $1.2 \times 10^{3}$. Compared with the polycrystalline thin film-based photodetector, the $I_{\text {dark }}$ in this study is higher. This is understandable since the asfabricated PNWs are characterized by few grain boundaries which fail to suppress the dark current $[11,17]$. In addition, photodetectors assembled from some other $\mathrm{PNW}$ arrays, including $\mathrm{MAPbBr}_{3}, \mathrm{MAPbCl}_{3}, \mathrm{FAPbBr}_{3}$, $\mathrm{FAPbCl}_{3}, \mathrm{CsPbI}_{3}$ and $\mathrm{Cs}_{3} \mathrm{Cu}_{2} \mathrm{I}_{5}$, have also exhibited welldefined photoresponses (Fig. S7).

The responsivity $(R)$ of the as-fabricated photodetector was evaluated and can be given by $R=I_{\mathrm{ph}} / P S$, where $S$ is the effective device area [45]. $R$ is calculated to be $20.56 \mathrm{~A} \mathrm{~W}^{-1}$ (net photocurrent $I_{\mathrm{ph}} \approx 2.3 \mathrm{nA}, S \approx$ $5 \times 10^{-5} \mathrm{~cm}^{2}$ ) under $520 \mathrm{~nm}$ light illumination (incident light power density: $2.2 \mu \mathrm{W} \mathrm{cm}{ }^{-2}$ ). According to the cal- culated value of $R$, the specific detectivity $\left(D^{*}\right)$ for the PNW arrays-based device is calculated to be $4.73 \times$ $10^{12}$ Jones by using the equation $D^{*}=R S^{1 / 2} /\left(2 e I_{\text {dark }}\right)^{1 / 2}$, where $e$ is the electron charge $\left(1.6 \times 10^{-19} \mathrm{C}\right)$ [46]. Table 1 lists $R$ and $D^{*}$ of the present device and other $\mathrm{MAPbI}_{3}-$ based photodetectors. It is seen that while the specific detectivity is comparable to other NWs-based devices, the responsivity is higher than most of devices listed in the table $[18,30,31]$, except for the one composed of NWs derived from fluid-guided antisolvent vapor-assisted crystallization method [22]. Such relatively good device performance is related to the reduced grain boundary and defects in the obtained PNW arrays. Both $R$ and $D^{*}$ are found to decrease as power density increases (Fig. 4e), indicative of intensified recombination loss at relatively high intensity [19]. In addition, the external quantum efficiency (EQE), defined as the ratio between the number of photoexcited electron-hole pairs and the number of incident photons, has been calculated according to the equation $\mathrm{EQE}=(h c R / e \lambda) \times 100 \%$, where $h$ is the Planck constant, $c$ and $\lambda$ represent the speed of light and the incident light wavelength, respectively [45]. The value of EQE reaches a maximum $4.89 \times 10^{3} \%$ (see Fig. S8), which means that internal gain plays a significant role in such a photoconductive device. Although it tends to decrease with increasing power density, it exceeds $1000 \%$ under all 
Table 1 Comparison of the performance between our photodetector and other $\mathrm{MAPbI}_{3}$-based devices

\begin{tabular}{|c|c|c|c|c|}
\hline Device structure & Measurement conditions & $R\left(\mathrm{~A} \mathrm{~W}^{-1}\right)$ & $D^{*}$ (Jones) & Ref. \\
\hline $\mathrm{MAPbI}_{3} \mathrm{MW}$ & $V=-5 \mathrm{~V} ; \lambda=420 \mathrm{~nm}, 1 \mu \mathrm{W} \mathrm{cm}{ }^{-2}$ & 13.57 & $5.25 \times 10^{12}$ & {$[31]$} \\
\hline $\mathrm{MAPbI}_{3} \mathrm{NW}$ & $V=1 \mathrm{~V} ; \lambda=650 \mathrm{~nm}, 4 \mu \mathrm{W} \mathrm{cm}$ & 0.85 & $2.5 \times 10^{12}$ & {$[30]$} \\
\hline $\mathrm{MAPbI}_{3} \mathrm{NW}$ & $V=1 \mathrm{~V} ; \lambda=530 \mathrm{~nm}, 2.5 \mathrm{nW} \mathrm{cm}{ }^{-2}$ & 4.95 & $2 \times 10^{13}$ & {$[18]$} \\
\hline $\mathrm{MAPbI}_{3} \mathrm{NW}$ & $V=5 \mathrm{~V} ; \lambda=550 \mathrm{~nm}, 0.1 \mu \mathrm{W} \mathrm{cm}{ }^{-2}$ & $1.25 \times 10^{4}$ & $1.73 \times 10^{11}$ & {$[22]$} \\
\hline $\mathrm{MAPbI}_{3} \mathrm{NW}$ & $V=1 \mathrm{~V} ; \lambda=520 \mathrm{~nm}, 2.2 \mu \mathrm{W} \mathrm{cm}^{-2}$ & 20.56 & $4.73 \times 10^{12}$ & Our work \\
\hline
\end{tabular}

tested conditions. To further unveil the dependence of responsivity on the wavelength of the incident light, normalized spectral response of the photodetector was recorded in the wavelength range of $200-1000 \mathrm{~nm}$ at a fixed power density $\left(50 \mu \mathrm{W} \mathrm{cm}{ }^{-2}\right)$. As we can see from Fig. 4f, the device exhibits a broadband photoresponse in the visible light region, but relatively weak photoresponse for wavelengths longer than $800 \mathrm{~nm}$, which is consistent with previously reported results $[18,22]$.

A number of $1 \mathrm{D}$ semiconductor nanostructures have demonstrated promising application for polarizationsensitive photodetectors, due to their anisotropic optical absorption arising from the high aspect ratio and oriented crystallization $[47,48]$. To further explore the possibility of current well-aligned $\mathrm{MAPbI}_{3} \mathrm{NW}$ arrays for polarization-dependent detection application, the optoelectronic property was then studied by shining a polarized light that is generated using a polarizer and can be continually rotated to change the polarization angle of the incident light. Apparently, the photocurrent exhibits a strong dependence on the polarization angle (Fig. 5b, c). The maximum photocurrent emerges with the polarization angle of $0^{\circ}$ when the polarization direction is parallel to the axial direction of the PNW. With the increase of the polarization angle, the photocurrent gradually decreases until it achieves a minimum value at the polarization angle of $90^{\circ}$. Further increase of polarization angle leads to a continuous increase in photocurrent until the po-
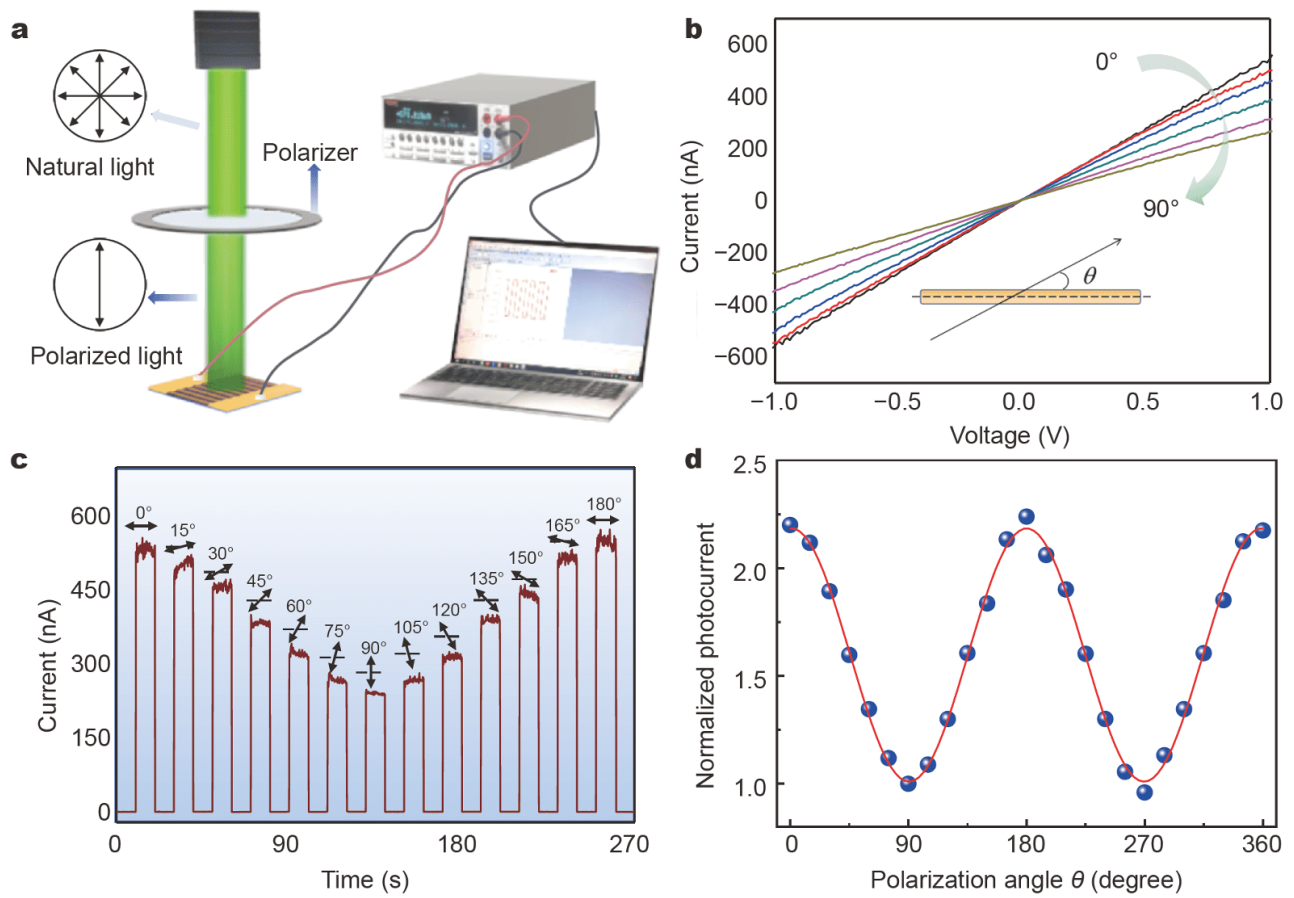

Figure 5 Application in the photodetection of polarized light. (a) Schematic diagram of measurement configuration for the polarization photodetection. (b) $I-V$ curves under incident light with different polarization angles. The polarization angle $(\theta)$ is defined as the angle between the incident light polarized direction and the axial direction of the PNW arrays. (c) Time-dependent photoresponses under incident light with different polarization angles. (d) Normalized photocurrent as a function of the polarization angle $\theta$. The unity corresponds to the photocurrent with the polarization angle of $90^{\circ}$. 
larization angle reaches $180^{\circ}$. Normalized polarizationdependent photocurrent is depicted in Fig. $5 \mathrm{~d}$, which exhibits a decent cosine wave and illustrates a polarization ratio of 2.2. This value is higher than that of oleic acid-passivated single-crystalline $\mathrm{MAPbI}_{3} \mathrm{NWs}_{\text {(1.3) [18] }}$ and comparable to those of inorganic single-crystalline $\mathrm{CsPbBr}_{3}$ NWs (2.6) [19] and $\beta$-CsPbI ${ }_{3}$ NWs (2.68) [44].

\section{CONCLUSIONS}

In summary, we have demonstrated a DVD-R-assisted NCG strategy for the facile and versatile fabrication of large-scale well-aligned PNW arrays with preferential crystallographic orientation by directly growing the perovskite precursor within the nanochannel. Without the assistance of a traditional photolithography process, this strategy shows excellent compatibility which allows the controlled growth of various PNWs on both rigid and flexible substrates. Further analysis reveals that the photodetector based on $\mathrm{MAPbI}_{3} \mathrm{NW}$ arrays exhibits obvious photoresponse properties with a responsivity of $20.56 \mathrm{~A} \mathrm{~W}^{-1}$ and specific detectivity of $4.73 \times 10^{12}$ Jones upon $520 \mathrm{~nm}$ illumination, respectively, which are much better than previously reported values. This relatively good optoelectronic performance should be attributed to the high-quality crystallinity and the highly oriented $1 \mathrm{D}$ nanostructure which provide an effective transport pathway for the photogenerated carriers. Meanwhile, the PNW array photodetector is also very sensitive to polarization light, with a polarization ratio of 2.2 . The proposed NCG strategy proves to be a cost-efficient and effective method for fabrication of high-quality PNW arrays with potential applications in integrated optoelectronic devices and systems.

Received 19 January 2021; accepted 22 February 2021; published online 28 April 2021

1 Ling Y, Yuan Z, Tian Y, et al. Bright light-emitting diodes based on organometal halide perovskite nanoplatelets. Adv Mater, 2016, 28: 305-311

2 Tan ZK, Moghaddam RS, Lai ML, et al. Bright light-emitting diodes based on organometal halide perovskite. Nat Nanotech, 2014, 9: 687-692

3 Lin P, Chen H, Wei Z, et al. Continuous-flow synthesis of doped all-inorganic perovskite nanocrystals enabled by a microfluidic reactor for light-emitting diode application. Sci China Mater, 2020, 63: 1526-1536

4 Zhou H, Chen Q, Li G, et al. Interface engineering of highly efficient perovskite solar cells. Science, 2014, 345: 542-546

5 Grätzel M. The light and shade of perovskite solar cells. Nat Mater, 2014, 13: 838-842

6 Green MA, Ho-Baillie A, Snaith HJ. The emergence of perovskite solar cells. Nat Photon, 2014, 8: 506-514
7 Liu Z, Ono LK, Qi Y. Additives in metal halide perovskite films and their applications in solar cells. J Energy Chem, 2020, 46: 215228

8 Hu X, Zhang X, Liang L, et al. High-performance flexible broadband photodetector based on organolead halide perovskite. Adv Funct Mater, 2014, 24: 7373-7380

9 Xue M, Zhou H, Xu Y, et al. High-performance ultraviolet-visible tunable perovskite photodetector based on solar cell structure. Sci China Mater, 2017, 60: 407-414

10 Feng J, Gong C, Gao H, et al. Single-crystalline layered metalhalide perovskite nanowires for ultrasensitive photodetectors. Nat Electron, 2018, 1: 404-410

11 Lian Z, Yan Q, Lv Q, et al. High-performance planar-type photodetector on (100) facet of $\mathrm{MAPbI}_{3}$ single crystal. Sci Rep, 2015, 5: 16563

12 Lee MM, Teuscher J, Miyasaka T, et al. Efficient hybrid solar cells based on meso-superstructured organometal halide perovskites. Science, 2012, 338: 643-647

13 Stranks SD, Eperon GE, Grancini G, et al. Electron-hole diffusion lengths exceeding 1 micrometer in an organometal trihalide perovskite absorber. Science, 2013, 342: 341-344

14 Xing G, Mathews N, Sun S, et al. Long-range balanced electronand hole-transport lengths in organic-inorganic $\mathrm{CH}_{3} \mathrm{NH}_{3} \mathrm{PbI}_{3}$. Science, 2013, 342: 344-347

15 Stoumpos CC, Malliakas CD, Kanatzidis MG. Semiconducting tin and lead iodide perovskites with organic cations: phase transitions, high mobilities, and near-infrared photoluminescent properties. Inorg Chem, 2013, 52: 9019-9038

16 Hodes G. Perovskite-based solar cells. Science, 2013, 342: 317-318

17 Cao F, Tian W, Wang M, et al. Semitransparent, flexible, and selfpowered photodetectors based on ferroelectricity-assisted perovskite nanowire arrays. Adv Funct Mater, 2019, 29: 1901280

18 Gao L, Zeng $\mathrm{K}$, Guo J, et al. Passivated single-crystalline $\mathrm{CH}_{3} \mathrm{NH}_{3} \mathrm{PbI}_{3}$ nanowire photodetector with high detectivity and polarization sensitivity. Nano Lett, 2016, 16: 7446-7454

19 Feng J, Yan X, Liu Y, et al. Crystallographically aligned perovskite structures for high-performance polarization-sensitive photodetectors. Adv Mater, 2017, 29: 1605993

20 Chen G, Feng J, Gao H, et al. Stable $\alpha-\mathrm{CsPb}_{3}$ perovskite nanowire arrays with preferential crystallographic orientation for highly sensitive photodetectors. Adv Funct Mater, 2019, 29: 1808741

$21 \mathrm{Gu}$ L, Poddar S, Lin Y, et al. A biomimetic eye with a hemispherical perovskite nanowire array retina. Nature, 2020, 581: 278282

22 Deng W, Huang L, Xu X, et al. Ultrahigh-responsivity photodetectors from perovskite nanowire arrays for sequentially tunable spectral measurement. Nano Lett, 2017, 17: 2482-2489

23 Wang S, Jiang Y, Juarez-Perez EJ, et al. Accelerated degradation of methylammonium lead iodide perovskites induced by exposure to iodine vapour. Nat Energy, 2017, 2: 16195

24 Chou SS, Swartzentruber BS, Janish MT, et al. Laser direct write synthesis of lead halide perovskites. J Phys Chem Lett, 2016, 7: 3736-3741

25 Alias MS, Dursun I, Shi D, et al. Focused-ion beam patterning of organolead trihalide perovskite for subwavelength grating nanophotonic applications. J Vacuum Sci Tech B, 2015, 33: 051207

$26 \mathrm{Gu} \mathrm{Z}$, Wang K, Li H, et al. Direct-writing multifunctional perovskite single crystal arrays by inkjet printing. Small, 2017, 13: 1603217

27 Lee W, Lee J, Yun H, et al. High-resolution spin-on-patterning of 
perovskite thin films for a multiplexed image sensor array. Adv Mater, 2017, 29: 1702902

28 Lee L, Baek J, Park KS, et al. Wafer-scale single-crystal perovskite patterned thin films based on geometrically-confined lateral crystal growth. Nat Commun, 2017, 8: 15882

29 Spina M, Bonvin E, Sienkiewicz A, et al. Controlled growth of $\mathrm{CH}_{3} \mathrm{NH}_{3} \mathrm{PbI}_{3}$ nanowires in arrays of open nanofluidic channels. Sci Rep, 2016, 6: 19834

30 Deng H, Dong D, Qiao K, et al. Growth, patterning and alignment of organolead iodide perovskite nanowires for optoelectronic devices. Nanoscale, 2015, 7: 4163-4170

31 Deng W, Zhang X, Huang L, et al. Aligned single-crystalline perovskite microwire arrays for high-Performance flexible image sensors with long-term stability. Adv Mater, 2016, 28: 2201-2208

32 Jeong B, Hwang I, Cho SH, et al. Solvent-Assisted gel printing for micropatterning thin organic-inorganic hybrid perovskite films. ACS Nano, 2016, 10: 9026-9035

33 Liu P, He X, Ren J, et al. Organic-inorganic hybrid perovskite nanowire laser arrays. ACS Nano, 2017, 11: 5766-5773

34 Kunkelmann C, Ibrahem K, Schweizer N, et al. The effect of threephase contact line speed on local evaporative heat transfer: Experimental and numerical investigations. Int J Heat Mass Transfer, 2012, 55: 1896-1904

35 Li SX, Xu YS, Li CL, et al. Perovskite single-crystal microwire-array photodetectors with performance stability beyond 1 year. Adv Mater, 2020, 32: 2001998

$36 \mathrm{Yu}$ W, Li F, Yu L, et al. Single crystal hybrid perovskite field-effect transistors. Nat Commun, 2018, 9: 5354

37 Levchuk I, Osvet A, Tang X, et al. Brightly luminescent and colortunable formamidinium lead halide perovskite $\mathrm{FAPbX}_{3}(\mathrm{X}=\mathrm{Cl}, \mathrm{Br}$, I) colloidal nanocrystals. Nano Lett, 2017, 17: 2765-2770

38 Chen H, Pina JM, Yuan F, et al. Multiple self-trapped emissions in the lead-free halide $\mathrm{Cs}_{3} \mathrm{Cu}_{2} \mathrm{I}_{5}$. J Phys Chem Lett, 2020, 11: 43264330

39 Kim A, Jang KS, Kim J, et al. Solvent-free directed patterning of a highly ordered liquid crystalline organic semiconductor via template-assisted self-assembly for organic transistors. Adv Mater, 2013, 25: 6219-6225

40 Hassanin H, Mohammadkhani A, Jiang K. Fabrication of hybrid nanostructured arrays using a PDMS/PDMS replication process. Lab Chip, 2012, 12: 4160-4167

41 Duan X, Huang Y, Cui Y, et al. Indium phosphide nanowires as building blocks for nanoscale electronic and optoelectronic devices. Nature, 2001, 409: 66-69

42 Agarwal R, Lieber CM. Semiconductor nanowires: optics and optoelectronics. Appl Phys A, 2006, 85: 209-215

43 Kind $\mathrm{H}$, Yan $\mathrm{H}$, Messer B, et al. Nanowire ultraviolet photodetectors and optical switches. Adv Mater, 2002, 14: 158-160

44 Zhou Y, Luo J, Zhao Y, et al. Flexible linearly polarized photodetectors based on all-inorganic perovskite $\mathrm{CsPbI}_{3}$ nanowires. Adv Opt Mater, 2018, 6: 1800679

45 Choi MS, Qu D, Lee D, et al. Lateral $\mathrm{MoS}_{2} \mathrm{p}-\mathrm{n}$ junction formed by chemical doping for use in high-performance optoelectronics. ACS Nano, 2014, 8: 9332-9340

46 Choi W, Cho MY, Konar A, et al. High-detectivity multilayer $\mathrm{MoS}_{2}$ phototransistors with spectral response from ultraviolet to infrared. Adv Mater, 2012, 24: 5832-5836

47 Wang J, Gudiksen MS, Duan X, et al. Highly polarized photoluminescence and photodetection from single indium phosphide nanowires. Science, 2001, 293: 1455-1457
Singh A, Li X, Protasenko V, et al. Polarization-sensitive nanowire photodetectors based on solution-synthesized CdSe quantum-wire solids. Nano Lett, 2007, 7: 2999-3006

Acknowledgements This work was supported by the National Natural Science Foundation of China (NSFC, 62074048), the Fundamental Research Funds for the Central Universities (PA2020GDKC0014, JZ2020HGTB0051, and JZ2018HGXC0001), and the Open Foundation of Anhui Provincial Key Laboratory of Advanced Functional Materials and Devices (4500-411104/011).

Author contributions Luo LB supervised the project; Huang R conceived the idea and carried out the sample fabrication and device measurements; Liu JY prepared the PDMS template and precursor solutions; Huang $\mathrm{R}$ and Lin $\mathrm{DH}$ conducted the optical, XRD, AFM and SEM characterizations; Huang R, Wu CY and Luo LB co-wrote the paper. $\mathrm{Wu} \mathrm{D}$ provided guidance in the experiments and characterizations. All the authors discussed the results, commented on and revised the manuscript.

Conflict of interest The authors declare that they have no conflict of interest.

Supplementary information Supplementary information and supporting data are available in the online version of the paper.

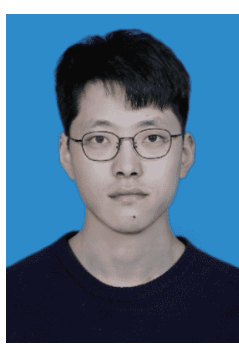

Rui Huang received his Bachelor degree from Hefei University of Technology, China. He is currently a graduate student at the School of Electronic Sciences and Applied Physics, Hefei University of Technology, China. His main research interest focuses on optoelectronic devices based on perovskite materials.

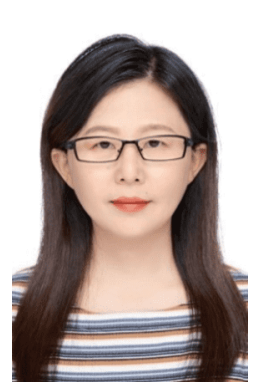

Chun-Yan Wu received her $\mathrm{PhD}$ degree in inorganic chemistry from the University of Science and Technology of China in 2006. She is currently a professor at the School of Electronic Science and Applied Physics, Hefei University of Technology, Hefei, China. Her research interests involve 2D layered semiconductors, photoelectronic devices and applications of photodetector arrays for integrated optoelectronic systems. 


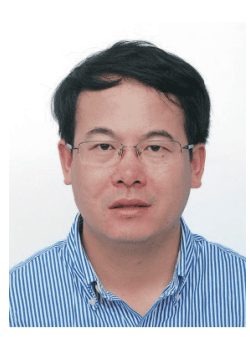

Lin-Bao Luo received his $\mathrm{PhD}$ degree from the Department of Physics and Materials Sciences, City University of Hong Kong under the guidance of Prof. Shuit-Tong Lee in 2009. After spending one and half years in the same group as a research associate, he joined the School of Electronic Sciences and Applied Physics, Hefei University of Technology, where he is now a full professor of applied physics. His research interest mainly focuses on high-performance optoelectronic and electronic device applications including photodetectors (UV light, NIR), photovoltaic devices, and nonvolatile memory devices etc.
纳米通道限域生长制备取向生长的钻针矿纳米线 阵列及其在偏振光探测方面的应用

黄瑞 ${ }^{1}$, 林笛婳 ${ }^{1}$, 刘佳音 ${ }^{1}$, 吴春艳 ${ }^{1^{*}}$, 吴翟 ${ }^{2}$, 罗林保 ${ }^{1 *}$

摘要 有序排列、取向生长的钙钛矿纳米线阵列的合成通常需要 复杂的工艺条件和精密的仪器设备, 这不利于其广泛应用. 本文提 出了一种纳米通道限域生长的方法来制备䥻钠矿纳米线阵列. 利 用场发射扫描电子显微镜、原子力显微镜和X射线衍射等分析手 段, 表征了纳米线阵列的形貌和晶体结构, 证明了该方法可用于在 各种衬底上制备大面积的具有取向生长特点的不同种类的钙钛矿 纳米线阵列. 基于 $\mathrm{MAPbI}_{3}$ 纳米线阵列所制备的光电探测器光响应 优异, 响应度和比探测率分别为 $20.56 \mathrm{~A} \mathrm{~W}^{-1}$ 和 $4.73 \times 10^{12}$ Jones. 此外, 由于所制备的一维钙钛矿纳米线结构的各向异性, 该光电探 测器还实现了对于偏振光的探测, 其偏振比为 2.2 . 